
mulheres

\title{
Socioeconomic characteristics, self-evaluation of health, and quality of life in women
}

\section{Características socioeconómicas, autoevaluación de salud y calidad de vida en mujeres}

\author{
Recebido: 13/12/2019 \\ Aprovado: 01/04/2010 \\ Publicado: 15/05/2020
}

\author{
Juliana Martins Pinto ${ }^{1}$ \\ Ana Paula Gomes Fernandes ${ }^{2}$ \\ Mariana Thays Carvalho ${ }^{3}$ \\ Cristiane Vitaliano Graminha ${ }^{4}$ \\ Adriana Cristina de Araújo Figueiredo 5 \\ Isabel Aparecida Porcatti de Walsh ${ }^{6}$
}

O objetivo foi investigar as relações entre características socioeconômicas, autoavaliação de saúde e qualidade de vida em mulheres. Trata-se de estudo transversal de base populacional com amostra de 1489 mulheres residentes em Uberaba, Minas Gerais, em 2014. As mulheres mais jovens (18-40 anos) foram frequentes no grupo "insatisfeitas com a saúde" $(\mathrm{p}<0,001)$, assim como aquelas com mais anos de escolaridade ( 9 anos ou mais) ( $\mathrm{p}=0,002)$ e as insatisfeitas com a renda $(\mathrm{p}<0,001)$. As mulheres entre 4159 anos $(p<0,001)$, com maior escolaridade $(p<0,001)$ e com renda insuficiente $(p<0,001)$ avaliaram a qualidade de vida como ruim com mais frequência do que as mulheres em outros grupos $(p<0,001)$. Qualidade de vida ruim e insatisfação com a saúde foram mais frequentes entre mulheres mais jovens, naquelas com maior escolaridade e insatisfeitas com a renda familiar.

Descritores: Saúde da mulher; Fatores socioeconômicos; Saúde Pública.

The objective of this research was investigation the relations between the socioeconomic features, health evaluation and quality of life in women. This is a cross-sectional study with a population bases and a sample of 1489 women living in Uberaba, Minas Gerais, in 2014. The youngest women (18-40) were frequent in the group "dissatisfied with health" ( $<<0.001)$, as were those with higher educational levels ( 9 years or more) $(\mathrm{p}=0.002)$ and those dissatisfied with their income $(\mathrm{p}<0.001)$. Women from 41$59(p<0.001)$, with higher educational levels $(p<0.001)$ and insufficient income $(p<0.001)$ evaluated their quality of life as bad more frequently than women in other groups $(\mathrm{p}<0.001)$. A bad quality of life and dissatisfaction with health were more frequent among younger women, those with lower educational levels, and those dissatisfied with their family income.

Descriptors: Women's health; Socioeconomics factors; Public Health.

El objetivo fue investigar las relaciones entre características socioeconómicas, autoevaluación de salud y calidad de vida en mujeres. Se trata de un estudio transversal de base poblacional con muestra de 1489 mujeres residentes en Uberaba, Minas Gerais, en 2014. Las mujeres más jóvenes (18-40 años) fueron comunes en el grupo "insatisfechas con la salud" $(\mathrm{p}<0,001)$, así como aquellas con más años de escolaridad ( 9 años o más) ( $\mathrm{p}=0,002)$ y las insatisfechas con los ingresos $(\mathrm{p}<0,001)$. Las mujeres entre 41-59 años $(\mathrm{p}<0,001)$, con mayor escolaridad $(\mathrm{p}<0,001)$ y con ingresos insuficientes $(\mathrm{p}<0,001)$ evaluaron la calidad de vida como mala con más frecuencia que las mujeres en otros grupos $(\mathrm{p}<0,001)$. Mala calidad de vida e insatisfacción con la salud fueron más comunes entre mujeres más jóvenes, en aquellas con mayor escolaridad e insatisfechas con los ingresos familiares.

Descriptores: Salud de la mujer; Factores socioeconómicos; Salud Pública.

* Estudo financiado pela Fundação de Amparo à Pesquisa de Minas Gerais - FAPEMIG, sob APQ-01825-12.

1. Fisioterapeuta. Pós Doutora em Fisioterapia e Epidemiologia do Envelhecimento. Professora do curso de Graduação em Fisioterapia na Universidade Federal Triângulo Mineiro (UFTM), Uberaba, MG, Brasil. ORCID: 0000-0003-2617-3308 E-mail: ju_fisio33@yahoo.com.br

2. Fisioterapeuta. Especialista em Fisioterapia na Saúde da Mulher. Mestranda do Programa de Pós-Graduação em Fisioterapia (PPGF) pela UFTM/ Universidade Federal de Uberlândia (UFU), Uberaba, MG, Brasil. ORCID: 0000-0001-5424-755X E-mail: ana-paulagf@gmail.com

3. Fisioterapeuta. Mestranda do PPGF/UFTM/UFU, Uberaba, MG, Brasil. ORCID: 0000-0003-0469-8226 E-mail: mary13_carvalho@hotmail.com

4. Fisioterapeuta. Doutora em Ciências Médicas. Professor Associado do curso de Graduação em Fisioterapia da UFTM, Uberaba, MG, Brasil. ORCID: 0000-0002-7013-2688. E-mail: crisgraminha@yahoo.com.br

5. Biomédica. Especialista em Análises Clínicas e Toxicologia. Aluna especial do PPGF/UFTM/UFU, Uberaba, MG, Brasil. ORCID: 0000-0002-3185-3482 E-mail: adriana.araujo@uftm.edu.br

6. Fisioterapeuta. Doutora em Fisioterapia. Professora Associada do curso de Graduação em Fisioterapia da UFTM e do PPGF da UFTM/UFU, Uberaba, MG, Brasil. ORCID: 0000-0002-2317-1326. E-mail: isabelpwalsh@gmail.com 


\section{INTRODUÇÃO}

A

s trajetórias de saúde e bem-estar na população brasileira tendem a ser diferentes dependendo das características econômicas, demográficas e sociais que determinam condições favoráveis ou não ao longo da vida ${ }^{1,2}$. Esses aspectos influenciam a carga de doenças e incapacidades, o uso e o acesso aos serviços de saúde, e a adesão aos tratamentos e programas de prevenção e promoção da saúde ${ }^{3,4}$.

A população feminina difere da população masculina quanto às condições de saúde e bem-estar, e as desigualdades tendem a aumentar com o avançar da idade ${ }^{5-7}$. As mulheres são mais longevas, apresentam maior número de doenças crônicas e incapacidades devido à menor exposição a acidentes, violência e outras causas externas de morte quando comparadas aos homens ${ }^{8}$, além de perceberem a própria saúde mais negativamente ${ }^{9}$. Esse cenário destaca a importância de compreender os fenômenos relacionados à saúde da mulher e as particularidades de seus processos de saúde-doença-incapacidade, para assim, melhorar a atenção à saúde dessa população.

Nos contextos epidemiológico e demográfico atuais, em que se observa aumento da incidência e prevalência de doenças crônicas não transmissíveis (DCNT), maior inserção da mulher no mercado de trabalho, mudanças nas estruturas familiares e dos papeis sociais, os indicadores subjetivos de saúde e bem-estar proporcionam avaliações mais eficientes, uma vez que fornecem informações relevantes sobre o quanto os eventos de saúde e de vida afetam o indivíduo ${ }^{1,5,10}$. Esse tipo de avaliação tem se destacado na saúde coletiva nos últimos anos, sendo reconhecida como paradigma de saúde atual11. Entre esses indicadores estão a autoavaliação de saúde e a autoavaliação da qualidade de vida.

A qualidade de vida é definida como a autoavaliação que o sujeito faz da própria condição de vida, considerando suas expectativas e realizações pessoais, bem como as exigências e padrões do contexto sociocultural ${ }^{12}$. 0 conceito tem sido amplamente investigado desde o início da transição do modelo biomédico de saúde para os modelos biopsicossocial, ecológico e holístico, que afirmam a importância e as possibilidades de manter uma vida com qualidade, apesar das doenças e adversidades dos ciclos de vida ${ }^{13}$. Desde então, a promoção de melhor qualidade de vida para as populações tem sido um dos principais desafios da sociedade atual, dos gestores e dos serviços de saúde.

A autoavaliação de saúde diz respeito à avaliação que o sujeito faz da própria saúde de maneira global, geralmente considerando o estado de saúde dos seus pares - comparação social e a comparação com si mesmo, o que há algum tempo chamava-se comparação pessoal10,14. Essa avaliação reflete o impacto das doenças, problemas de saúde, sintomas e incapacidades na vida do indivíduo, especialmente sobre sua independência e autonomia. Tal conceito tem sido fortemente relacionado com piores prognósticos funcionais e de saúde e com maior mortalidade ${ }^{15}$.

Diversas condições ou eventos podem ocorrer ao longo da vida e predispor as pessoas à baixa qualidade de vida e pior autoavaliação de saúde. Doenças, incapacidade, perda de autonomia, avanço da idade, acidentes, problemas financeiros, relações familiares estressantes e falta de apoio social são os mais comuns ${ }^{16,17}$.

Algumas condições levam à diminuição da qualidade de vida e da saúde e se caracterizam por serem pouco ou nada mutáveis, o que coloca o indivíduo em desvantagem prévia perante seus pares. Essas desigualdades podem estar presentes entre as mulheres, determinando diferentes desfechos em saúde e qualidade de vida. Tais condições incluem idade, escolaridade, renda e convívio com um companheiro em união estável.

0 conhecimento sobre as diferenças socioeconômicas relacionadas à qualidade de vida e autoavaliação de saúde de mulheres contribui para compreender a extensão desses fenômenos diante de desigualdades e desvantagens da população feminina. Desse modo, o objetivo deste estudo foi investigar as relações entre características socioeconômicas, autoavaliação de saúde e qualidade de vida em mulheres. 


\section{MÉTODO}

Os dados são oriundos do Inquérito de Saúde da Mulher - ISA, realizado em Uberaba, Minas Gerais, em 2014. Trata-se de estudo transversal de base populacional, com amostra probabilística de 1556 mulheres com idade igual ou superior a 18 anos, residentes no município. 0 projeto foi aprovado pelo Comitê de Ética em Pesquisa da Universidade Federal do Triângulo Mineiro, sob o CAAE no 1826/2010 e financiado pela Fundação de Amparo à Pesquisa do Estado de Minas Gerais (FAPEMIG), sob no APQ - 01825-12.

A amostragem deu-se de forma aleatória estratificada em dois estágios, considerandose os setores censitários e os domicílios, de acordo com o Censo/2010 do Instituto Brasileiro de Geografia e Estatística (IBGE). 0 critério de elegibilidade foi ter idade igual ou superior a 18 anos, residir em Uberaba-MG, compreender os objetivos da pesquisa e concordar em responder ao protocolo, mediante a assinatura do Termo de Consentimento Livre e Esclarecido (TCLE).

As entrevistas foram realizadas no domicílio, por entrevistadoras do sexo feminino, treinadas e devidamente identificadas, coordenadas por docentes, pesquisadores e alunos bolsistas. 0 treinamento dos pesquisadores compreendeu a aplicação dos questionários, palestras sobre noções e técnicas para entrevista, bem como estudo piloto com amostra de conveniência.

O conteúdo do protocolo de pesquisa foi elaborado especialmente para o estudo, mediante a seleção de instrumentos e medidas validadas para a população brasileira. 0 cálculo amostral considerou a ausência de conhecimento prévio sobre as estimativas das prevalências dos eventos de interesse, e, portanto, todos foram considerados iguais a $50 \%$. Considerou-se também um nível de confiança de 95\%, margem de erro de no máximo 2,5\% e $20 \%$ de perdas.

A Qualidade de Vida (QV) foi avaliada pela pergunta extraída do World Health Organization Questionnaire of Quality of Life (WHOQOL bref): "como você avalia sua qualidade de vida?", para a qual as opções de resposta foram muito ruim, ruim, mais ou menos, boa ou muito boa. Devido à baixa prevalência de qualidade de vida muito ruim, as categorias foram agrupadas para possibilitar as análises estatísticas. Sendo assim, as mulheres com qualidade de vida "muito ruim" e "ruim" foram categorizadas como tendo qualidade de vida "ruim" e aquelas cuja qualidade de vida era "boa" e "muito boa", como tendo qualidade de vida "boa". A categoria intermediária foi mantida conforme o instrumento original, e denominada "mais ou menos".

A autoavaliação de saúde foi indicada, também, por uma pergunta extraída do WHOQOL Bref: “Quão satisfeito você está com sua saúde?”, cujas opções de respostas foram: muito insatisfeita; insatisfeita; nem satisfeita nem insatisfeita; satisfeita e muito satisfeita. 0 agrupamento de categorias foi utilizado novamente para classificar as mulheres em "satisfeitas com a saúde" ou "insatisfeitas com a saúde".

As condições socioeconômicas investigadas foram faixa etária (18 a 40 anos; 41 a 59 anos; e 60 anos ou mais), satisfação com a renda, anos de escolaridade (nenhum; 1 a 4 anos; 5 a 8 anos; e 9 anos ou mais), e se vive com um companheiro em união estável. Todas as informações foram autorrelatadas.

Foram descritas as prevalências em frequência e porcentagem, e, posteriormente, as associações das variáveis socioeconômicas com autoavaliação de saúde e com qualidade de vida foram testadas pelo teste Qui-quadrado de Pearson e pelo Exato de Fisher, com significância de $5 \%$. As diferenças entre os grupos foram identificadas pela análise de resíduos ajustados padronizados $<1,96$. As análises foram realizadas no programa SPSS, versão 24.

\section{RESULTADOS}

0 valor estimado de mulheres a serem pesquisadas era de 1530, sendo entrevistadas 1556, mas considerou-se 1489, aquelas que apresentaram dados completos para os objetivos do presente estudo. 
A distribuição de mulheres nas faixas etárias 18-40, 41-59 e 60+ foi, respectivamente, de 32,9\%,35,9\% e 31,2\%. 0 percentual de mulheres que relataram ter frequentado a escola por menos de quatro anos foi $25,7 \% ; 48,2 \%$ das mulheres vivem sem companheiro e $72,4 \%$ declararam que sua renda não é suficiente para satisfazer as necessidades diárias (Tabela 1).

As comparações de proporções entre as mulheres satisfeitas e insatisfeitas com a saúde mostraram que as mulheres mais jovens (18-40 anos) são mais frequentes no grupo insatisfeitas $(\mathrm{p}<0,001)$, assim como aquelas que têm mais anos de escolaridade (9 anos ou mais) $(p=0,002)$ e aquelas que declararam insatisfação com a renda $(p<0,001)$. Não foi observada associação entre autoavaliação de saúde e união estável ( $p=0,541)$ (Tabela 1).

Tabela 1. Caracterização da amostra e associações entre variáveis socioeconômicas e autoavaliação de saúde (n=1489). Uberaba, MG. 2014.

\begin{tabular}{|c|c|c|c|c|}
\hline \multirow{2}{*}{ Variáveis } & \multicolumn{4}{|c|}{ Quão satisfeito você está com sua saúde? } \\
\hline & F (\%) & Satisfeita $(n=263)$ & Insatisfeita (n=1226) & p* \\
\hline \multicolumn{5}{|c|}{ Faixa etária } \\
\hline $18-40$ & $490(32,9)$ & $63(12,9)[24]$ & $427(87,1)[34,8]$ & \multirow{3}{*}{$<0,001$} \\
\hline $41-59$ & $534(35,9)$ & $119(22,3)[22,3]$ & $415(77,7)[33,8]$ & \\
\hline $60+$ & $465(31,2)$ & $81(17,4)[17,4]$ & $384(82,6)[31,3]$ & \\
\hline \multicolumn{5}{|c|}{ Anos de escolaridade } \\
\hline Nenhum & $55(3,9)$ & $13(23,6)[5,1]$ & $42(76,4)[3,6]$ & \multirow{4}{*}{0,002} \\
\hline $1-4$ & $309(21,8)$ & $67(21,7)[26,3]$ & $242(78,3)[20,8]$ & \\
\hline $5-8$ & $347(24,5)$ & $76(21,9)[29,8]$ & $271(78,1)[23,3]$ & \\
\hline $9+$ & $706(49,8)$ & $99(14)[38,8]$ & $607(86)[52,2]$ & \\
\hline \multicolumn{5}{|c|}{ União estável } \\
\hline Não & $717(48,2)$ & $122(17)[46,4]$ & $595(83)[48,6]$ & \multirow{2}{*}{0,541} \\
\hline Sim & $771(51,8)$ & $141(18,3)[53,6]$ & $630(81,7)[51,4]$ & \\
\hline \multicolumn{5}{|c|}{ Satisfação com a renda } \\
\hline Não & $1077(72,4)$ & $299(21,3)[87,4]$ & $848(78,7)[69,2]$ & \multirow{2}{*}{$<0,001$} \\
\hline Sim & $411(27,6)$ & $33(8)[12,6]$ & $378(92)[30,8]$ & \\
\hline
\end{tabular}

Com relação à qualidade de vida, as mulheres na meia-idade $(41-59$ anos $)(p<0,001)$, com maior escolaridade $(p<0,001)$ e com renda insuficiente $(p<0,001)$ avaliaram a qualidade de vida como ruim com mais frequência do que as mulheres em outros grupos ( $\mathrm{p}<0,001)$. Não foram observadas associações entre qualidade de vida e união estável $(p=0,716)$ (Tabela 2).

Tabela 2. Associações entre variáveis socioeconômicas e qualidade de vida. Uberaba, MG. 2014.

Como você avalia sua qualidade de vida?

\begin{tabular}{|c|c|c|c|c|}
\hline Variáveis $\mathbf{F}(\%)$ & $\begin{array}{c}\text { Ruim/muito ruim } \\
\text { (n=76) }\end{array}$ & $\begin{array}{c}\text { Mais ou menos } \\
\quad(n=362)\end{array}$ & $\begin{array}{c}\text { Boa/muito boa } \\
(n=1051)\end{array}$ & $\mathbf{p}$ \\
\hline \multicolumn{5}{|l|}{ Faixa etária ${ }^{-}$} \\
\hline $18-40$ & $15(3,1)[19,7]$ & $111(22,7)[30,7]$ & $364(74,3)[34,6]$ & \multirow{3}{*}{$<0,001$} \\
\hline $41-59$ & $38(7,1)[50]$ & $132(24,7)[36,5]$ & $364(68,2)[34,6]$ & \\
\hline $60+$ & $23(4,9)[30,3]$ & $119(25,6)[32,9]$ & $323(69,5)[30,7]$ & \\
\hline \multicolumn{5}{|c|}{ Anos de escolaridade $\underline{b}$} \\
\hline Nenhum & $4(7,3)[5,4]$ & $20(36,4)[5,8]$ & $31(56,4)[3,1]$ & \multirow{4}{*}{$<0,001$} \\
\hline $1-4$ & $20(6,5)[27]$ & $107(34,6)[31]$ & $182(58,9)[18,2]$ & \\
\hline $5-8$ & $22(6,3)[29,7]$ & $89(25,6)[25,8]$ & $236(68)[23,6]$ & \\
\hline $9+$ & $28(4)[37,8]$ & $129(18,3)[37,4]$ & $549(77,8)[55]$ & \\
\hline \multicolumn{5}{|l|}{ União estávelạ } \\
\hline Não & $37(5,2)[51,3]$ & $181(25,2)[50]$ & $499(69,6)[52,5]$ & \multirow{2}{*}{0,716} \\
\hline Sim & $39(5,1)[48,7]$ & $181(23,5)[50]$ & $551(71,5)[47,5]$ & \\
\hline \multicolumn{5}{|c|}{ Satisfação com a renda $a^{a}$} \\
\hline Não & $65(6)[85,5]$ & $301(27,9)[83,1]$ & $711(66)[67,7]$ & \multirow{2}{*}{$<0,001$} \\
\hline Sim & $11(2,7)[14,5]$ & $61(14,8)[16,9]$ & $3392,5)[32,3]$ & \\
\hline
\end{tabular}




\section{DISCUSSÃO}

Os resultados mostraram que existem desigualdades, principalmente em relação à idade, escolaridade e satisfação com a renda, demonstrando que os grupos mais sujeitos às percepções negativas de saúde e qualidade de vida são as mulheres mais jovens, com maior escolaridade e insatisfeitas com a renda.

Com exceção da insatisfação com a renda, esses resultados contrariam as hipóteses iniciais do estudo, construídas com base nas evidências sobre a crescente incidência de doenças crônicas que acompanham o processo de envelhecimento e os efeitos negativos dessas condições sobre a funcionalidade e, consequentemente, sobre o bem estar ${ }^{10}$. A premissa era de que pessoas que vivem em condições econômicas e sociais desfavoráveis, nesse caso, mulheres mais velhas ${ }^{18,19}$ e com menor escolaridade 20,21 , tendem a apresentar pior estado de saúde e funcionalidade, e, portanto, apresentariam piores avaliações subjetivas em relação à própria saúde e qualidade de vida, o que não foi confirmado por esse estudo.

Com relação à idade, um estudo ${ }^{10}$ afirmou que, à medida que a velhice avança, os problemas de saúde tornam-se mais estreitamente relacionados aos sintomas psicológicos, de modo que a depressão, por exemplo, aumenta a probabilidade de pior autoavaliação de saúde. Entretanto, o mesmo estudo encontrou que a relação entre pior saúde física e autoavaliação de saúde negativa é mais forte entre pessoas mais jovens quando comparadas com as mais velhas, provavelmente, devido à maior prevalência de depressão nos mais jovens ${ }^{10}$. Os achados da presente pesquisa, do mesmo modo, sugere a existência de variáveis mediadoras que expliquem a percepção negativa de saúde entre pessoas jovens.

Estudos mostraram que pessoas com baixa renda e baixa escolaridade apresentam piores avaliações subjetivas de saúde ${ }^{14,22}$, possivelmente porque tais condições implicam em maiores limitações físicas, que restringem a participação em atividades comunitárias, o acesso aos serviços de saúde, além de aumentarem o risco de depressão, incapacidades e morte ${ }^{21,23,24}$. Por outro lado, recursos pessoais e sociais podem ser recrutados frente às adversidades, permitindo aos indivíduos lidarem com os problemas e vivenciarem experiências positivas, mesmo perante condições desfavoráveis ${ }^{25,26}$. Tais recursos incluem resiliência, espiritualidade, suporte social e familiar, senso de autoeficácia e autonomia, seletividade socioemocional, entre outros $^{27,28 .}$.

A disponibilidade e a utilização desses recursos podem explicar, em parte, as observações empíricas que encontraram pessoas que relataram boa qualidade de vida e saúde, mesmo na presença de situações adversas ${ }^{19}$. Adicionalmente, pode haver influência da idade e da escolaridade na melhor percepção das condições reais de vida, de modo que pessoas mais jovens e com maior escolaridade podem ter maior acesso e compreensão das informações ao seu redor, e serem mais críticas e exigentes em relação às expectativas e potencialidades da própria existência e condições de vida, o que explica a baixa satisfação com a saúde e com a qualidade de vida entre as mulheres com maior escolaridade.

Pesquisas sugerem também que os idosos podem ter maior habilidade em lidar e superar eventos estressantes devido às experiências acumuladas ao longo da vida ${ }^{29,30}$. Também na velhice, evidencia-se o comportamento seletivo frente às adversidades, para garantir a preservação do estado emocional e do bem estar ${ }^{26}$. Essas explicações parecem ser mais adequadas para os resultados observados nesse estudo, apontando o envelhecimento como processo do curso de vida, em que saúde e qualidade de vida são determinadas por desigualdades sociais e econômicas.

\section{CONCLUSÃo}

A qualidade de vida ruim e a insatisfação com a saúde foram mais frequentes entre mulheres mais jovens e não entre as mais velhas, naquelas com maior escolaridade e não entre aquelas com baixa escolaridade, e entre as insatisfeitas com a renda familiar. 
Não foi observada relação com a convivência com ou sem companheiro. As relações entre características socioeconômicas, autoavaliação de saúde e qualidade de vida são complexas, podendo ser mediadas e moderadas por outras variáveis que denotam a disponibilidade de recursos psicossociais que determinariam, de fato, como as pessoas irão conviver e lidar com as adversidades e seus reflexos em seu bem-estar.

Os resultados do presente estudo foram obtidos por meio de métodos epidemiológicos reconhecidos que garantem a qualidade e a validade dos achados para a população de mulheres residentes em Uberaba-MG. Entretanto, a generalização ou extrapolação para outras populações deve ser cautelosa.

Embora as medidas autorrelatadas e subjetivas sejam úteis nos campos da Gerontologia e Saúde Coletiva, as limitações inerentes aos inquéritos populacionais, como a carência de medidas objetivas e específicas, podem levar à negligência de informações relevantes para a compreensão dos fenômenos investigados. Desse modo, mais estudos com outras variáveis são encorajados.

\section{REFERÊNCIAS}

1. Antunes JLF, Chiavegatto Filho ADP, Duarte YAO, Lebrão ML. Desigualdades sociais na autoavaliação de saúde dos idosos da cidade de São Paulo. Rev Bras Epidemiol. [Internet]. 2019 [citado em 03 fev 2020]; 21(2):e180010. Disponível em: http://www.scielo.br/pdf/rbepid/v21s2/1980-5497-rbepid-21-s2-e180010.pdf. $\quad$ DOI: 10.1590/1980-549720180010.supl.2

2. Tovar Cuevas LM, Perea Mosquera LN, Tovar Cuevas JR, Zúñiga Martínez CL. Determinantes sociales de la salud autorreportada: Colombia después de una década. Mundo Saúde [Internet]. 2018 [citado em 03 fev 2020]; 42(1):230-47. Disponível em: http://bvsms.saude.gov.br/bvs/periodicos/mundo_saude_artigos/determinantes_salud_colo mbia.pdfDOI: 10.15343/0104-7809.20184201230247

3. Louvison MCP, Lebrão ML, Duarte YAO, Santos JLF, Malik AM, de Almeida ES. Desigualdades no uso e acesso aos serviços de saúde entre idosos do município de São Paulo. Rev Saude Publ. [Internet]. 2008 [citado em 03 fev 2020]; 42(4):733-40. Disponível em: http://www.scielo.br/pdf/rsp/v42n4/6846.pdf

4. Barreto SM, Passos VMA, Giatti L. Comportamento saudável entre adultos jovens no Brasil. Rev Saude Públ. [Internet]. 2009 [citado em 03 fev 2020]; 43(2):9-17. Disponível em: http://www.scielo.br/pdf/rsp/v43s2/ao799.pdf

5. Carrapato P, Correia P, Garcia B. Determinante da saúde no Brasil: a procura da equidade na saúde. Saúde Soc. [Internet]. 2017 [citado em 03 fev 2020]; 26(3):676-89. Disponível em: http://www.scielo.br/pdf/sausoc/v26n3/0104-1290-sausoc-26-03-00676.pdf. $\quad$ DOI: 10.1590/S0104-12902017170304

6. Malta DC, Bernal RTI, Nunes ML, Oliveira MM, Iser BPM, Andrade SSCA, et al. Prevalência de fatores de risco e proteção para doenças crônicas não transmissíveis em adultos: estudo transversal, Brasil 2012. Epidemiol Serv Saúde [Internet]. 2014 [citado em 03 fev 2020]; 23(4):609-22. Disponível em: http://www.scielo.br/pdf/ress/v23n4/2237-9622-ress-23-0400609.pdf. DOI: 10.5123/S1679-49742014000400003

7. Pinto JM, Neri AL. Doenças crônicas, capacidade funcional, envolvimento social e satisfação em idosos comunitários: Estudo Fibra. Ciênc Saúde Colet. [Internet]. 2013 [citado em 03 fev 2020]; 18(12):3449-60. Disponível em: http://www.scielo.br/pdf/csc/v18n12/a02v18n12.pdf. DOI: http://dx.doi.org/10.1590/S1413-81232013001200002

8. Giacomin KC, Uchôa E, Firmo JOA, Lima-Costa MF. Projeto Bambuí: um estudo de base populacional da prevalência e dos fatores associados à necessidade de cuidador entre idosos. 
Cad Saúde Públ. [Internet]. 2005 [citado em 03 fev 2020]; 21(1):80-91. Disponível em: http://www.scielo.br/pdf/csp/v21n1/10.pdf. DOI: http://dx.doi.org/10.1590/S0102311X2005000100010

9. Pinto JM, Neri AL. Relationships between levels of social participation, self-rated health and life satisfaction in older adults according to gender. Rev Kairós [Internet]. 2016 [citado em 03 fev 2020]; 19(4):255-72. Disponível em: https://www.researchgate.net/publication/322316952

10. French DJ, Sargent-Cox K, Luszcz MA. Correlates of subjective health across the aging lifespan: understanding self-rated health in the oldest old. J Aging Health [Internet]. 2012 [citado em 03 fev 2020]; 24(8):1449-69. Disponível em: https://www.ncbi.nlm.nih.gov/pubmed/23103451. DOI: 10.1177/0898264312461151

11. Veras RP. Estratégias para o enfrentamento das doenças crônicas: um modelo em que todos ganham. Rev Bras Geriatr Gerontol. [Internet]. 2011 [citado em 03 fev 2020]; 14(4):779-86. Disponível em: http://www.scielo.br/pdf/rbgg/v14n4/a17v14n4.pdf. DOI: http://dx.doi.org/10.1590/S1809-98232011000400017

12. Fleck MPA. O instrumento de avaliação de qualidade de vida da Organização Mundial da Saúde (WHOQOL-100): características e perspectivas. Ciênc Saúde Colet. [Internet]. 2000 [citado em 03 fev 2020]; 5(1):33-8. Disponível em: http://www.scielo.br/pdf/csc/v5n1/7077.pdf. $\quad$ http://dx.doi.org/10.1590/S141381232000000100004

13. Ravenek MJ, Skarakis-Doyle E, Spaulding SJ, Jenkins ME, Doyle PC. Enhancing the conceptual clarity and utility of the international classification of functioning, disability \& health: the potential of a new graphic representation. Disabil Rehabil. [Internet]. 2012 [citado em 03 fev 2020]; 35:1-11. Disponível em: https://www.ncbi.nlm.nih.gov/pubmed/23072218. DOI: 10.3109/09638288.2012.717582

14. Borim FSA, Barros MBA, Neri A. Autoavaliação da saúde em idosos: pesquisa de base populacional no Município de Campinas, São Paulo, Brasil. Cad Saúde Públ. [Internet]. 2013 [citado em 03 fev 2020]; 28(4):769-80. Disponível em: http://www.scielo.br/pdf/csp/v28n4/16.pdf. DOI: http://dx.doi.org/10.1590/S0102311X2012000400016

15. Reile R, Stickley A, Leinsalu M. Large variation in predictors of mortality by levels of selfrated health: results from an 18-year follow-up study. Public Health [Internet]. 2017 [citado em 03 fev 2020]; 145:59-66. Disponível em: https://www.ncbi.nlm.nih.gov/pubmed/28359392. DOI: http://dx.doi.org/10.1016/j.puhe.2016.12.034

16. Mássimo EAL, Souza HNF, Freitas MIF. Chronic non-communicable diseases, risk and health promotion: social construction of Vigitel participants. Cienc Saúde Colet. [Internet]. 2015 [citado em 03 fev 2020]; 20(3):679-88. Disponível em: http://www.scielo.br/pdf/csc/v20n3/1413-8123-csc-20-03-00679.pdf. DOI: 10.1590/141381232015203.14742014

17. Pinto JM, Fontaine AM, Neri AL. The influence of physical and mental health on life satisfaction is mediated by self-rated health: a study with brazilian elderly. Arch Gerontol Geriatr. [Internet]. 2016 [citado em 03 fev 2020]; 65:104-10. Disponível em: https://www.ncbi.nlm.nih.gov/pubmed/27017415. DOI: 10.1016/j.archger.2016.03.009

18. Gana K, Bailly N, Saada Y, Joulain M, Alaphilippe D. Does life satisfaction change in old age: results from an 8-year longitudinal study. J Gerontol B Psychol Sci Soc Sci. [Internet]. 2012 [citado em 03 fev 2020]: 68(4):540-52. Disponível em: https://www.ncbi.nlm.nih.gov/pubmed/23103381. DOI: 10.1093/geronb/gbs093

19. Strawbridge WJ, Wallhagen MI, Cohen RD. Successful aging and well-being: self-rated compared with Rowe and Kahn. Gerontologist [Internet]. 2002 [citado em 03 fev 2020]; 42(6):727-33. Disponível em: https://www.ncbi.nlm.nih.gov/pubmed/12451153. DOI: 10.1093/geront/42.6.727 
20. Sousa ACPA, Guerra RO, Tu MT, Phillips SP, Guralnik JM, Zunzunegui MV. Life course adversity and physical performance across countries among men and women aged 65-74. PLoS One [Internet]. 2014 [citado em 03 fev 2020]; 9(8):1-10. Disponível em: https://journals.plos.org/plosone/article?id=10.1371/journal.pone.0102299. DOI: https://doi.org/10.1371/journal.pone.0102299

21. Erdem Ö, Van Lenthe FJ, Prins RG, Voorham TA, Burdorf A. Socioeconomic inequalities in psychological distress among urban adults: the moderating role of neighborhood social cohesion. PLoS One [Internet]. 2016 [citado em 03 fev 2020]; 11(6):e0157119. Disponível em: https://www.ncbi.nlm.nih.gov/pubmed/27280601. DOI: 10.1371/journal.pone.0157119

22. Loyola Filho AI, Firmo JOA, Uchôa E, Lima-Costa MF. Fatores associados à autoavaliação negativa da saúde entre idosos hipertensos e/ou diabéticos: resultados do projeto Bambuí. Rev Bras Epidemiol. [Internet]. 2013 [citado em 03 fev 2020]; 16(3):559-71. Disponível em: http://www.scielo.br/pdf/rbepid/v16n3/pt_1415-790X-rbepid-16-03-00559.pdf. $\quad$ DOI: http://dx.doi.org/10.1590/S1415-790X2013000300001

23. Syddall H, Evandrou M, Cooper C, Sayer AA. Social inequalities in grip strength, physical function, and falls among community dwelling older men and women: findings from the Hertfordshire Cohort Study. J Aging Health [Internet]. 2009 [citado em 03 fev 2020]; 21(6):91339. Disponível em: http://www.ncbi.nlm.nih.gov/pubmed/19597159. DOI: 10.1177/0898264309340793

24. García Ramírez JA, Vélez Álvarez C. Determinantes sociales de la salud y la calidad de vida en población adulta de Manizales, Colombia. Rev Cuba Salud Pública [Internet]. 2017 [citado em 03 fev 2020]; 43(2):191-203. Disponível em: http://scielo.sld.cu/pdf/rcsp/v43n2/spu06217.pdf

25. George LK. Still happy after all these years: research frontiers on subjective well-being in later life. J Gerontol B Psychol Sci Soc Sci. [Internet]. 2010 [citado em 03 fev 2020]: 65B(3):3319. Disponível em: https://www.ncbi.nlm.nih.gov/pubmed/20233742. DOI: 10.1093/geronb/gbq006

26. Scheibe S, Carstensen L. Emotional aging: recent findings and future trends. J Gerontol B Psychol Sci Soc Sci. [Internet]. 2010 [citado em 03 fev 2020]; 65B(2):135-44. Disponível em: https://www.ncbi.nlm.nih.gov/pmc/articles/PMC2821944/. DOI: 10.1093/geronb/gbp132

27. Landau R, Litwin H. Subjective well-being among the old-old: the role of health, personality and social support. Int J Aging Hum Dev. [Internet]. 2001 [citado em 03 fev 2020]; 52(4):26580. Disponível em: https://www.ncbi.nlm.nih.gov/pubmed/11474750. DOI: 10.2190/RUMTYCDX-X5HP-P2VH

28. Bennett KM, Reyes-Rodriguez MF, Altamar P, Soulsby LK. Resilience amongst older Colombians living in poverty: an ecological approach. J Cross Cult Gerontol. [Internet]. 2016 [citado em 03 fev 2020]; 31(4):385-407. Disponível em: https://www.ncbi.nlm.nih.gov/pubmed/27585577. DOI: http://dx.doi.org/10.1007/s10823016-9303-3

29. Fontes AP, Neri AL. Resilience in aging: literature review. Cienc Saude Colet [Internet]. 2015 [citado em 03 fev 2020]; 20(5):1475-95. Disponível em: http://www.ncbi.nlm.nih.gov/pubmed/26017950. 10.1590/141381232015205.00502014

30. Steptoe A, Deaton A, Stone AA. Subjective wellbeing, health, and ageing. Lancet [Internet]. 2014 [citado em 03 fev 2020]; 385(9968):640-8. Disponível em: http://linkinghub.elsevier.com/retrieve/pii/S0140673613614890. DOI: $10.1016 / S 0140-$ 6736(13)61489-0 


\section{CONTRIBUIÇÕES}

Shamyr Sulyvan de Castro e Isabel Aparecida Porcatti de Walsh contribuíram na concepção, análise e interpretação dos dados, redação e revisão. Adriana Cristina de Araújo Figueiredo, Juliana Martins Pinto, Ana Paula Gomes Fernandes, Mariana Thays Carvalho e Cristiane Vitaliano Graminha participaram na análise e interpretação dos dados, redação e revisão.

\section{Como citar este artigo (Vancouver)}

Pinto JM, Fernandes APG, Carvalho MT, Graminha CV, Figueiredo ACA, Walsh IAP. Características socioeconômicas, autoavaliação de saúde e qualidade de vida em mulheres. REFACS [Internet]. 2020 [citado em inserir dia, mês e ano de acesso]; 8(2):210-218. Disponível em: inserir link de acesso. DOI: inserir link do DOI

\section{Como citar este artigo (ABNT)}

PINTO, J. M.; FERNANDES, A. P. G.; CARVALHO, M. T.; GRAMINHA, C. V.; FIGUEIREDO, A. C. A. REFACS, Uberaba, MG, v. 8, n. 2, p. 210-218, 2020. Disponível em: inserir link de acesso. Acesso em: inserir dia, mês e ano de acesso. DOI: inserir link do DOI.

\section{Como citar este artigo (APA)}

Pinto, J. M., Fernandes, A. P. G., Carvalho, M. T. C., Graminha, C. V., Figueiredo, A. C., Walsh, I. A. P. (2020). Características socioeconômicas, autoavaliação de saúde e qualidade de vida em mulheres. REFACS, 8(2), 210-218. Recuperado em: inserir dia, mês e ano de acesso de inserir link de acesso. DOI: inserir link do DOI. 